\title{
Developing a curriculum in artificial intelligence for emergency radiology
}

\author{
Edmund M. Weisberg ${ }^{1}$ (D) Elliot K. Fishman ${ }^{2}$ \\ Published online: 10 July 2020 \\ (C) American Society of Emergency Radiology 2020
}

In the short term, it is likely that COVID-19 is much more prevalent in your thinking than what insight into artificial intelligence (AI) might help your career or what a course in deep learning (DL) and AI might look like in medical schools in the next few years. We understand and acknowledge the significant stress the pandemic is placing on all of us and extend our heartfelt appreciation to those of our colleagues in emergency and infectious disease medicine as well as researchers that toil with great effort to mitigate the public health crisis.

\section{Short-term expectations and plans}

It is difficult to know what life will look like after we have managed to corral COVID-19, but it is rational to expect that there will be significant societal shifts. Telecommuting would seem to be much more likely, and the rapid escalation in telemedicine in the early days of the pandemic will probably endure. While much else can be speculated on, it is reasonable to assume that the use of AI will become further embedded in medicine.

Whether working in isolation or not, we suspect that the radiology community itself has moved beyond the debate as to whether AI will impact the field. We know that it will. What remains questionable or debatable is to what degree or at what depth it will affect radiology. That is difficult to predict. Regardless, even with a minimal impact, we know that AI will play an active role in diagnostic radiology.

Edmund M. Weisberg and Elliot K. Fishman contributed equally to this work.

Edmund M. Weisberg

eweisbe1@jhmi.edu

1 Johns Hopkins Medicine, 601 North Caroline Street, JHOC 3262, Baltimore, MD 21287, USA

2 Johns Hopkins Hospital, Baltimore, MD, USA
This will likely occur in the next 3 to 5 years. It takes longer than that to train residents. In the meantime, current residents are not learning anything meaningful about AI. At this point, however, is there interest in such training and, if so, do we know just what should be taught on the subject?

\section{Interest in incorporating $\mathrm{Al}$ in radiology training}

A 2018 anonymous survey of a single radiology residence training program, with 69 of $104(66 \%)$ trainees and attending radiologists responding, revealed that $29 \%(n=12)$ claimed to have used AI during their daily work. Trainees were more likely to learn about AI but also more likely to question whether they would have opted for diagnostic radiology as a specialty had they known the probability of the impact of AI. These results suggest the need to provide some pedagogical guidance on the uses of AI for radiology trainees [1]. Further, one result gleaned from another anonymous online survey that year, in this case across all Canadian medical schools, was that students were especially interested in "expert opinions on AI" and "discussing AI in preclinical radiology lectures," suggesting that they were not getting this kind of exposure in their training [2].

The next year, Pinto Dos Santos et al. reported on a webbased anonymous questionnaire distributed to students at three major medical schools intended to assess radiology students' attitudes toward AI use in their field. Of the 263 respondents, more than two thirds $(71 \%)$ expressed the belief that AI should be included in medical training [3]. In response to these findings, Gallix and Chong, in writing on the use of AI in radiology, endorsed the notion that the principles and application of AI should be taught in medical school [4]. Late last year, an online questionnaire was directed to radiologists, students, and surgeons in Switzerland's German-speaking medical community. Of the 170 individuals responding, 55 were students, who expressed greater skepticism on the use of AI than radiologists, viewing it as a potential threat to diagnostic radiologists [5]. 
Does such concern expressed in this latter study represent a roadblock in incorporating AI into the curriculum for radiology training? We think that a more recent finding is likely more indicative of future interest and trends. An anonymous survey with 484 responses from 19 UK medical schools was reported early in 2020 , revealing that $89 \%$ of respondents expressed the belief that learning AI would benefit them in their careers, $78 \%$ supported training in $\mathrm{AI}$ as part of the medical curriculum, while $49 \%$ noted feeling less inclined toward radiology as a specialty because of the influence of AI [6].

The most significant takeaway from these studies may be that a need to clarify the role of AI in radiology is called for, and interest in developing curricula in AI for radiology training programs is slowly emerging [7]. But how will it take shape? Or how will we shape it? Journals like Emergency Radiology and societies like the American Society of Emergency Radiology (ASER) have the opportunity to play important roles in defining a roadmap.

\section{What should radiology trainees learn about Al?}

Working AI education into the curriculum might help dispel any lingering doubts among students and any radiologists harboring concerns. Are there core common principles and standards that we can identify for such a curriculum?

At this point, it should be considered significant that there are at least $25 \mathrm{AI}$ apps approved by the FDA for use in diagnostic radiology, in the emergency setting, with approximately 15 now available for sale. Practicing radiologists, trainees, and technicians would all benefit from keeping up to date on the use of these new products.

A pre-curricular approach to identifying directions and content for AI should consider the following:

- Early understanding of how AI is affecting and may further affect the field.

- A survey of the literature covering the first uses of AI in radiology, particularly emergency radiology.

- Trainees should get a sense of how convoluted AI is through an interdisciplinary focus even though they do not likely have any expertise in computer science.

- A survey of the available AI apps, which will likely be in the triple figures by the time a rudimentary curriculum is implemented.

- Realistic use cases and limitations of AI, including potential hacking or abuses of AI.
There is no perfect answer or clear academic program ready to delineate. The technology is evolving rapidly, and even the literature that might be suitable now will be obsolete or less compelling in just a couple of years. But we should start with something. Such a rollout should begin with a course or two, based largely on a survey of the current literature and the current usage of the approved apps. At some point not far down the line, a comprehensive education policy to address $\mathrm{AI}$ in radiology may be appropriate, including fuller learning modules that include surveys into the complexity of deep learning and further exploration into app development and expanding the use of AI beyond the emergency radiology realm. Curriculum development will also have to acknowledge some of the inevitable changes in the healthcare system and society at large as a result of our response to the COVID19 global pandemic. Nevertheless, the Emergency Room and Emergency Radiology seem like the ideal spots to begin the transition of AI/DL into our practice. We can hardly wait.

\section{Compliance with ethical standards}

Conflict of interest The authors declare that they have no conflict of interest.

\section{References}

1. Collado-Mesa F, Alvarez E, Arheart K (2018) The role of artificial intelligence in diagnostic radiology: a survey at a single radiology residency training program. J Am Coll Radiol 15(12):1753-1757

2. Gong B, Nugent JP, Guest W, Parker W, Chang PJ, Khosa F, Nicolaou S (2019) Influence of artificial intelligence on Canadian medical students' preference for radiology specialty: a National Survey Study. Acad Radiol 26(4):566-577

3. Pinto Dos Santos D, Giese D, Brodehl S, Chon SH, Staab W, Kleinert R et al (2019) Medical students' attitude towards artificial intelligence: a multicenter survey. Eur Radiol 29(4):1640-1646

4. Gallix B, Chong J (2019) Artificial intelligence in radiology: who's afraid of the big bad wolf? Eur Radiol 29(4):1637-1639

5. van Hoek J, Huber A, Leichtle A, Härmä K, Hilt D, von TenggKobligk H, Heverhagen J, Poellinger A (2019) A survey on the future of radiology among radiologists, medical students and surgeons: students and surgeons tend to be more skeptical about artificial intelligence and radiologists may fear that other disciplines take over. Eur J Radiol 121:108742

6. Sit C, Srinivasan R, Amlani A, Muthuswamy K, Azam A, Monzon L, Poon DS (2020) Attitudes and perceptions of UK medical students towards artificial intelligence and radiology: a multicenter survey. Insights Imaging 11(1):14

7. Tajmir SH, Alkasab TK (2018 Jun) Toward augmented radiologists: changes in radiology education in the era of machine learning and artificial intelligence. Acad Radiol 25(6):747-750

Publisher's note Springer Nature remains neutral with regard to jurisdictional claims in published maps and institutional affiliations. 